References: 1. Lund-Johansen P: Hemodynamic changes at rest and during exercise in long-term prazosin therapy for essential hypertension, in Prazosin Clinical Symposium Proceedings. Published as a special report by Postgraduate Medicine. New York, McGraw.
Hill Co, 1975, pp 45-52. 2. Komajda M. Prazosin and lipids: A study of the effects of prazosin Hili Co, 1975, pp 45-52.2. Komajda M: Prazosin and lipids: A study of the eftects of prazzosien
on blood lipids in hypertensive patients. Impact int December 1986; (speciat issue): 1-4 3. Rouffy J, Jaillard J: Effects of two antihypertensive agents on lipids, lipoproteins, an apoproteins A and B. Comparison of prazosin and atenolol, Am J Med 1986,80(supp

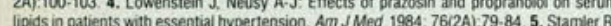
Stamler $J$ Gosch $F C$, et at: Initial antihypertensive drug therapy. A comparison of alph blocker (prazosin) and diuretic (hydrochlorothiazide): Final report of a randomized, con trolled trial. In press. 6. Leren P. Helgeland A, Holme I, et al: Effect of propranolol and prazosin on blood lipids: The Osio Study. Lancet 1980; II:4-6. 7. Kwan CM. Shepherd AMM. Johnson J. et al: Forearm and finger hemodynamics, blood pressure control and lipid changes in patients with diabetic hypertension treated with atenolol and prazosin. Clin
Pharmacol Ther 1988,44:202-209. 8. Leichter SB. Baumgardner B: Eftects of chronic prazosin therapy on intermediary metabolism in diabetic patients. $J$ Cardiovasc Med 1981.(special suppl): $38-42$

Briel Summary

For Oral Use

NOICATIONS AND USAGE: MINIPRESS (prazosin hydrochloride) is indicated in the treat ment of hypertension. It is mild to moderate in activity and can be used as the initial agent on in a general treatment program in conjunction with a diuretic and/or other antihypertensive
drugs as needed. CONTRAINDICATIONS: None known. WARNINGS: MINIPRESS may cause syncope with sudden loss of consciousness. In most cases this is believed to be due to an excessive postural hypotensive effect, although occasionally the syncopa episode has been preceded by a bout of severe tachycardia with heart rates of $120-16 \mathrm{C}$ beats per minute. Syncopal episodes have usually occurred within 30 to 90 minutes of the initial dose of the drug: occasionally they have been reported in association with rapic dosage increases or the introduction of another antihypertensive drug into the regimen 0 approximately $1 \%$ in patients given an initial dose of $2 \mathrm{mg}$ or greater. Clinical trials conducted during the investigational phase of this drug suggest that syncopal episodes conducted during the investigational phase of this drug suggest that syncopal episodes
can be minimized by limiting the initial dose of the drug to $1 \mathrm{mg}$. by subsequently increasing the dosage slowly, and by introducing any additional antiliypertensive drug: into the patient's regimen with caution (see DOSAGE AND ADMINISTRATION). Hypoten sion may develop in patients given MINIPRESS who are also receiving a beta-blocke such as propranolol. If syncope occurs, the patient should be placed in the recumbent position and treated supportively as necessary. This adverse effect is sell-limiting and in most cases does not recur after the initial period of therapy or during subsequent dose titration. The patient should also be cautioned to avoid situations where injury could resull Should syncope occur during the initiation of MINIPRESS therapy. PRECAUTIONS: Informa tion for Patients: Diziness or drowsiness may occur after the first dose of this medicine Avoid driving or performing hazardous tasks for the first 24 hours after taking this medicine or when the dose is increased. Dizziness, lightheadedness or tainting may occur, especially Then fising from a lying or sitting position. Getting up slowly may help lessen the problem if the weather is hot. While taking MINIPRESS, be careful in the amount of alcohol you drink Also, use extra care during exercise or hot weather, or it standing for long periods. Check with your physician if you have any questions. Drug Interactions: MiNIPRESS has been administered without any adverse drug interaction in limited clinical experience to date with the following: (1) cardiac glycosides-digitalis and digoxin; (2) hypoglycemics-insulin, chiorpropamide. phenformin, tolazamide, and tolbutamide; (3) tranquilizers and sedatives-

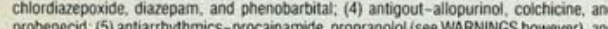
probenecid, (5) antiarthythmics-procainamide, propranolor (see WARNINGS however), and indomethacin, and phenylbutazone. Addition of a diuretic or other antihypertensive, aspenin MINIPRESS has been shown to cause an additive hivpotensive effect. Orug/Laboratory Jes Interactions: False positive results may occur in screening tests for pheochromocytoma in
patients who are being treated with prazosin. If an levated VMA is found, prazosin should be discontinued and the patient retested after a month. Laboratory Tests: In clinical studies in which lipid profiles were followed, there were generally no adverse changes noted between pre-and post-treatment lipid levels. Carcinogenesis, Mutagenesis, Impairment of Fer. tility: No carcinogenic potential was demonstrated in an 18 month study in rats with MINIPRESS (prazosin hydrochloride) at dose levels more than 225 times the usual maximum recommended human dose of $20 \mathrm{mg}$ per day. MINIPRESS was not mutagenic in in vivo genetic toxicology studies. In a fertility and general reproductive performance study in rats both males and temales, treated with $75 \mathrm{mg} / \mathrm{kg}$ (225 times the usual maximum recom-
mended human dose), demonstrated decreased fertility while those treated with $25 \mathrm{mg} / \mathrm{kg}$ 75 times the usual maximum recommended human dose) did not. In chronic studies year or more) of MINIPRESS in rats and dogs, testiculas changes consisting of atrophy an necrosis occurred at $25 \mathrm{mg} / \mathrm{kg} / \mathrm{day}$ (75 times the usual maximum recommended hum an dose). No testicular changes were seen in rats or dogs at $10 \mathrm{mg} / \mathrm{kg} / \mathrm{day}$ ( 30 times the usua maximum recommended human dose). In view of the testicular changes observed in animals, 105 patients on long term MINIPRESS therapy were monitored for 17-ketosteroid excretion and no changes indicating a drug effect were observed. In addition, 27 males on MINIPRESS tor up to 51 months did not have changes in sperm morphology suggestive o drug effect. Usage in Pregnancy: Pregnancy Category C. There are no adequate and weil controlled studies which establish the safety of MiviPRESS (prazosin $\mathrm{HC}$ ) in pregnant The potential risk to she mould be used during pregnancy only if the potential benefit justifie: be excreted in small amounts in human milk. Caution should be exercised when MiNiPPESS is administered to a nursing win human milk. Caution should be exercised when MINIPARESS have not been established. ADVFRSE REACTIONS: Clinical trials were conducted on more than 900 patients. During these trials and subsequent marketing experience, the most trequent reactions associated with MINIPRESS therapy are: dizziness $10.3 \%$, headache
7.896 , drowsiness $7.69 \%$, lack of energy $6.9 \%$, weakness $6.5 \%$, palpitations $5.39 \%$, and 7.8\% . drowsiness $7.69 \%$, lack of energy $6.99 \%$, weakness $6.5 \%$, palpitations $5.3 \%$, and nausea $4.9 \%$. In most instances side effects have disappeared with continued therapy or have been tolerated with no decrease in dose of drug. Less frequent adverse reactions which are reported to occur in 1-4\% of patients are: Gastrointestinal: vomiting, diarnhea, constipa tion, Cardiovascular: edema, orthostatic hypotension, dyspnea, syncope; Central Nervous
System, vertigo, depression, nervousness; Dermatologic: rash; Genitourinary: urinary freSystem: vertigo, depression, nervousness; Dermatologic: rash; Genitourinary urinary fre-
quency. EENT. blurred vision, reddened sclera, epistaxis, dry mouth, nasal congestion. In addition, fewer than $1 \%$ of patients have reported the foliowing (in some instances, exact causal relationships have not been established): Gastrointestinat: abdominal discomfor and/or pain, liver function abnormalities, pancreatitis; Cardiovascular tachycardia; Central planus, Genitourinary incontinence, impotence, priapism, EENT: tinnitus; Other: diaphoresis, fever. Single reports of pigmentary mottling and serous retinopathy. and a few reports cataract development or disappearance have been reported. OVERDOSAGE: Should overdosage lead to hypotension, support of the cardiovascular system is of first importance.
Restoration of blood pressure and normalization of heart rate may be accomplished by Restoration of blood pressure and normalization of heart rate may be accomplished by
keeping the patient in the supine position. If this measure is inadequate, shock should first be keeping the patient in the supine position. If this measure is inadequate, shock should firstbe
treated with volume expanders. If necessary, vasopressors should then be used. Renal function should be monitored and supported as needed. Laboratory data indicate The dose of MINIPRESS should be adjusted according to individual blood pressure response. Initial Dose: $1 \mathrm{mg}$ two or three times a day. Maintenance Dose: Dosage may
resure response. Initial Dose: $1 \mathrm{mg}$ two or three times a day. Maintenance Dose: Dosage may
be stowly increased to a total daily dose of $20 \mathrm{mg}$ given in divided doses. The therapeutic dosages most commonly employed have ranged from $6 \mathrm{mg}$ to $15 \mathrm{mg}$ daily given in divided doses. Doses higher than $20 \mathrm{mg}$ usually do not increase efficacy: however a few patients may benefit from further increases up to a daily dose of $40 \mathrm{mg}$ given in divided
doses. After initial titration some patients can be maintained adequately on a twice daily dosage regimen. Use With Other Drugs: When adding a diuretic or other antihypertensive agent, the dose of MiNIPRESS should be reduced to $1 \mathrm{mg}$ or $2 \mathrm{mg}$ three times a day and retitration then carried out. Hevised December 1988

alty, he should be commended. If he is not totally satisfied with his situation, then perhaps Dr Baron should reevaluate his goals.

Clearly, Dr Baron misunderstood one of my concluding statements, "Many hospitals need house officers and would probably welcome such a solution to their personnel shortages." I was not suggesting that osteopathic residents, or any other residents for that matter, be put in any institution simply as warm bodies to do "scut work." What I meant to convey was that hospitals without training programs would be very willing, I believe, to fund training programs. Such financial support would enable the osteopathic profession to institute highly, qualified academic training programs in larger hospitals with a significant number of diverse patients. Having been a resident once myself, I certainly do not advocate the "service residency" type of program. Likewise, residents are not around to do "scut work."

Concerning Dr Karp's kind comments, I would like to thank and commend him for his suggestion that the profession must rise above individual concerns to maintain the strength of osteopathic medicine. In my article, I suggested that a great deal of time and effort must be spent in evaluating the problems related to our residency programs. It may take 5 or 10 years before we can approach these problems with an intelligent plan. In order to do this, I believe that the profession, including academically qualified individuals, must come together under the auspices of the American Osteopathic Asso- ciation and work toward a solution.

One thing is certain: Many of our residency programs are strictly service-oriented. We must dispense with this type of training program and introduce very structured, educational, and exciting programs for our residents.

ANDREW A. PECORA, DO Professor and Chairman Department of Medicine University of Medicine and Dentistry of New Jersey -School of Osteopathic Medicine

Stratford, NJ

\section{Commenting on L- tryptophan and osteopathic physicians in allopathic residencies}

\section{To the Editor:}

In response to Dr Northup's editorial "Natural is not always healthy" (JAOA 1990;90:505), it should have been obvious early on that something other than Ltryptophan was causing eosinophilia-myalgia syndrome (EMS). The Centers for Disease Control has traced this EMS outbreak to one foreign manufacturer of Ltryptophan, which implies contamination.

I do not see such an outcry over drugs used in medicine that often cause deleterious effects. For example, chemotherapeutic agents are known to produce additional malignancies in as many as $25 \%$ of the patients receiving such treatment. Natural may not always be healthy, as Dr (continued on page 759) 


\section{Another}

therapy with confidence for

\section{gastric}

patients with a history of ulcer:*

ulcer-free

Only Cytotec replaces the

NSAID

naturally protective prostaglandins

patient

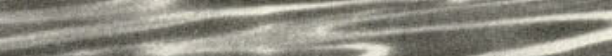

that NSAIDs deplete.

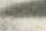

(4)

- 40
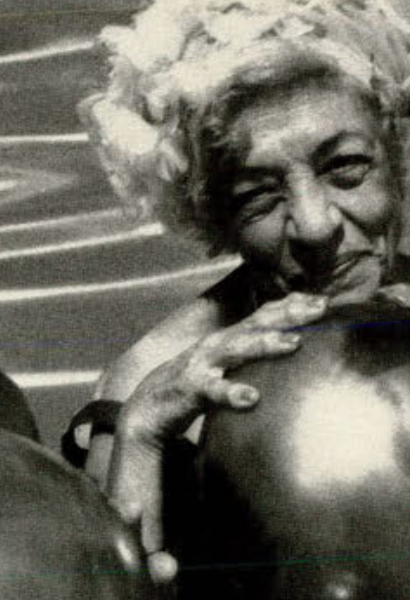

a.

,

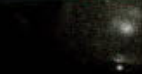

a.
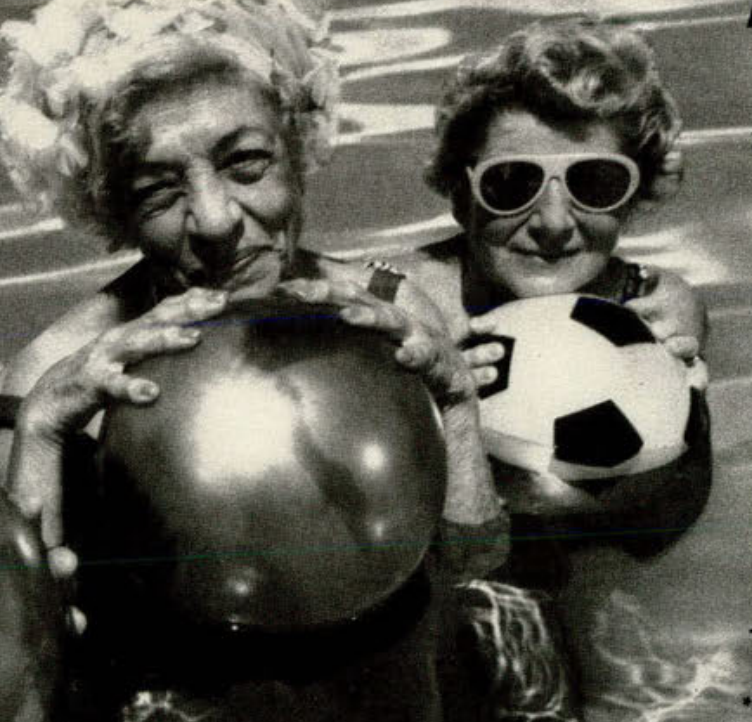

हi.

gens:

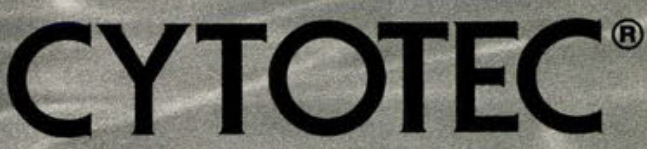

(misonrostol) $\begin{aligned} & 200-m c g \\ & \text { tablets }\end{aligned}$

Confidence to prescribe NSAIDs

for patients with a history of ulcer

*Cytotec is indicated for patients at high risk of developing gastric ulceration, such as those with a history of ulcer, and patients at high risk of complications from gastric ulcer, $\mathrm{gg}$ the elderly and those with concomitant debilitating disease.

Please see adjoining page for brief summary of prescribing information.

\section{SEARLE}




\section{СYTOTЕС

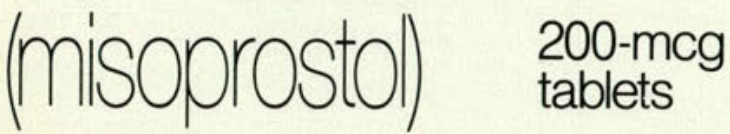

\section{Considerations before you prescribe Cytotec}

- Because of its abortifacient property, Cytotec should not be prescribed for women who are pregnant. Patients must be advised of the abortifacient property and warned not to give the drug to others.
Cytotec should be used in a woman of childbearing potential only if she is using effective contraceptive measures, has received oral and written warnings concerning the hazards of misoprostol, has had a negative serum pregnancy test within two weeks prior to beginning therapy, and will begin therapy only on the second or third day of the next menstrual period.

Some patients may experience transient diarrhea, which usually resolves in about a week, or abdominal discomfort. Abdominal discomfort may persist in the absence of gastric ulceration.

\section{BRIEF SUMMARY}

CONTRAINDICATIONS AND WARNINGS: Cytotec (misoprostol) is contraindicated, because of its abortifacient property, in women who are pregnant. (See Precautions.) Patients must be advised of the abortifacient property and warned Precautions.)
not to give the drug to others. Cytotec should not be used in women of childbearing potential unless the patient requires nonsteroidal anti-inflammatory drug (NSAID) therapy and is at high risk of complications from gastric ulcers associated with use of the NSAID, or is at high risk of developing gastric ulceration. In such patients. Cytotec may be prescribed if the patient

- is capable of complying with effective contraceptive measures.

- has received both oral and written warnings of the hazards of misoprostol, the risk of possible contraception failure, and the danger to other women of childbearing potential should the drug be taken by mistake.

- has had a negative serum pregnancy test within two weeks prior to beginning therapy.

- will begin Cytotec only on the second or third day of the next normal menstrua period.

INDICATIONS AND USAGE: Cytotec (misoprostol) is indicated for the prevention of NSAID (nonsteroidal anti-inflammatory drugs, including aspirin)-induced gastric ulcers in patients at high risk of complications from gastric ulcer, eg. the elderly and patients with concomitant debilitating disease, as well as patients at high risk of developing gastric ulceration, such as patients with a history of ulcer CYytotec has developing gastric ulceration, such as patients with a history of ulcer. Cytotec has
not been shown to prevent duodenal ulcers in patients taking NSAIDs. Cytotec should be taken for the duration of NSAID therapy. Cytotec has been shown to preven gastric ulcers in controlled studies of three months' duration. It had no effect, compared to placebo, on gastrointestinal pain or discomfort associated with NSAID use.

CONTRAINDICATIONS: SEe bOXed CONTRAINDICATIONS AND WARNINGS

Cytotec should not be taken by anyone with a history of allergy to prostaglandins. WARNINGS: See boxed CONTRAINDICATIONS AND WARNINGS.

\section{PRECAUTIONS:}

Information for patients: Cytotec is contraindicated in women who are pregnant, and should not be used in women of childbearing potential unless the patient requires nonsteroidal anti-inflammatory drug (NSAID) therapy and is at high risk of complications from gastric ulcers associated with the use of the NSAID, or is at high risk of developing gastric ulceration. Women of childbearing potential should be told that they must not be pregnant when Cytotec therapy is initiated, and that they must use an effective contraception method while taking Cytotec.

See boxed CONTRAINDICATIONS AND WARNINGS.

Patients should be advised of the following:

Cytotec is intended for administration along with nonsteroidal anti-inflammatory drugs (NSAIDs), including aspirin, to decrease the chance of developing an NSAIDinduced gastric ulcer.

Cytotec should be taken only according to the directions given by a physician.

If the patient has questions about or problems with Cytotec, the physician should be contacted promptly.

THE PATIENT SHOULD NOT GIVE CYTOTEC to ANYONE ELSE. Cytotec has been prescribed for the patient's specific condition, may not be the correct treatment for another person, and may be dangerous to the other person if she were to become pregnant.

The Cytotec package the patient receives from the pharmacist will include a leaflet containing patient information. The patient should read the leaflet before taking Cytotec and each time the prescription is renewed because the leaflet may have been revised.

Keep Cytotec out of the reach of children.

SPECIAL NOTE FOR WOMEN: Cytotec must not be used by pregnant women. Cytotec may cause miscarriage. Miscarriages caused by Cytotec may be incomplete, which could lead to potentially dangerous bleeding, hospitalization, surgery, infertility, or maternal or fetal death.

Drug interactions: See Clinical Pharmacology. Cytotec has not been shown to interfere with the beneficial effects of aspirin on signs and symptoms of rheumatoid arthritis. Cytotec does not exert clinically significant effects on the absorption, blood levels, and antiplatelet effects of therapeutic doses of aspirin. Cytotec has no clinically significant effect on the kinetics of diclofenac or ibuprofen.

Animal toxicology: A reversible increase in the number of normal surface gastric epithelial cells occurred in the dog, rat, and mouse. No such increase has been observed in humans administered Cytotec for up to one year.

An apparent response of the female mouse to Cytotec in long-term studies at 100 to 1000 times the human dose was hyperostosis, mainly of the medulla of sternebrae. Hyperostosis did not occur in long-term studies in the dog and rat and has not been seen in humans treated with Cytotec.

Carcinogenesis, mutagenesis, impairment of fertility: There was no evidence of an effect of Cytotec on tumor occurrence or incidence in rats receiving daily doses up to 150 times the human dose for 24 months. Similarly, there was no effect of Cytotec on tumor occurrence or incidence in mice receiving daily doses up to 1000 times the human dose for 21 months. The mutagenic potential of Cytotec was tested in several in vitro assays, all of which were negative.

Misoprostol, when administered to breeding male and female rats at doses 6.25 times to 625 times the maximum recommended human therapeutic dose, produced dose-related pre- and post-implantation losses and a significant decrease in the number of live pups born at the highest dose. These findings suggest the possibility of a general adverse effect on fertility in males and females.

Pregnancy: Pregnancy Category $\mathrm{X}$. See boxed CONTRAINDICATIONS AND WARNINGS

Nonteratogenic effects: Cytotec may endanger pregnancy (may cause miscarriage) and thereby cause harm to the fetus when administered to a pregnant woman. Cytotec produces uterine contractions, uterine bleeding, and expulsion of the products of conception. Miscarriages caused by Cytotec may be incomplete. In studies in women undergoing elective termination of pregnancy during the first trimester. Cytotec caused partial or complete expulsion of the products of conception in $11 \%$ of the subjects and increased uterine bleeding in $41 \%$. If a woman is or becomes pregnant while taking this drug, the drug should be discontinued and the patient apprised of the potential hazard to the fetus.

Teratogenic effects: Cytotec is not fetotoxic or teratogenic in rats and rabbits at doses 625 and 63 times the human dose, respectively.

Nursing mothers: See Contraindications. Cytotec should not be administered to nursing mothers because the potential excretion of misoprostol acid could cause significant diarrhea in nursing infants.

Pediatric use: Safety and effectiveness in children below the age of 18 years have not been established.

ADVERSE REACTIONS: The following have been reported as adverse events in subjects receiving Cytotec:

Gastrointestinal: The most frequent gastrointestinal adverse events were diarrhea and abdominal pain. The incidence of diarrhea ranged up to $40 \%$ but averaged $13 \%$ in clinical trials.

Diarrhea was dose related and usually developed early in the course of therapy (after 13 days), usually was self-limiting (often resolving after 8 days), but sometimes required discontinuation of Cytotec ( $2 \%$ of the patients). Rare instances of profound diarrhea leading to severe dehydration have been reported. Patients with an underlying condition such as inflammatory bowel disease, or those in whom dehydration, were it to occur, would be dangerous, should be monitored carefully if Cytotec is prescribed. The incidence of diarrhea can be minimized by administering after meals and at bedtime, and by avoiding coadministration of Cytotec with magnesium-containing antacids.

Gynecological: Women who received Cytotec during clinical trials reported the following gynecological disorders: spotting $(0.7 \%)$, cramps $(0.6 \%)$, hypermenorrhea $(0.5 \%)$, menstrual disorder $(0.3 \%)$ and dysmenorrhea $(0.1 \%)$. Postmenopausal vaginal bleeding may be related to Cytotec administration. If it occurs, diagnostic workup should be undertaken to rule out gynecological pathology.

Elderly: There were no significant differences in the safety profile of Cytotec in approximately 500 ulcer patients who were 65 years of age or older compared with younger patients.

Additional adverse events which were reported are categorized as follows: Incidence greater than 1\%: In clinical trials, the following adverse reactions were reported by more than $1 \%$ of the subjects receiving Cytotec and may be causally related to the drug: nausea $(3.2 \%)$, flatulence $(2.9 \%)$, headache $(2.4 \%)$, dyspepsia $(2.0 \%)$, vomiting $(1.3 \%)$, and constipation $(1.1 \%)$. However, there were no significant differences between the incidences of these events for Cytotec and placebo.

Causal relationship unknown: The following adverse events were infrequently reported. Causal relationships between Cytotec and these events have not been established but cannot be excluded: aches/pains, asthenia, fatigue, fever, rigors. weight changes, rash, dermatitis, alopecia, pallor, breast pain, abnormal taste, weight changes, rash, dermatitis, alopecia, pallor, breast pain, abnormal taste,
abnormal vision, conjunctivitis, deafness, tinnitus, earache, upper respiratory tract infection, bronchitis, bronchospasm, dyspnea, pneumonia, epistaxis, chest pain, edema, diaphoresis, hypotension, hypertension, arrhythmia, phlebitis, increased cardiac enzymes, syncope, Gl bleeding, GI inflammation/infection, rectal disorder, abnormal hepatobiliary function, gingivitis, reflux, dysphagia, amylase increase, glycosuria, gout, increased nitrogen, increased alkaline phosphatase, polyuria, dysuria, hematuria, urinary tract infection, anxiety, change in appetite, depression, drowsiness, dizziness, thirst, impotence, loss of libido, sweating increase, neuropathy, neurosis. arthralgia, myalgia, muscle cramps, stiffness, back pain, anemia, abnormal differential. thrombocytopenia, purpura, ESR increased.

Important note: Complete prescribing information should be consulted prior to use.

DOSAGE AND ADMINISTRATION: The recommended adult oral dose of Cytotec for the prevention of NSAID-induced gastric ulcers is $200 \mathrm{mcg}$ four times daily with food. If this dose cannot be tolerated, a dose of $100 \mathrm{mcg}$ can be used. Cytotec should be taken for the duration of NSAID therapy as prescribed by the physician Cytotec should be taken with a meal, and the last dose of the day should be at bedtime.

Renal impairment: Adjustment of the dosing schedule in renally impaired patients is not routinely needed, but dosage can be reduced if the $200-\mathrm{mcg}$ dose is not tolerated

Address medical inquires to:

$1 / 23 / 90 \cdot P 90-R 114 \mathrm{~V}$

G.D. Searle \& Co.

Medical \& Scientific Information Department

4901 Searle Parkway

Skokie IL 60077

SERLE G.D. Searle \& Co. 


\section{The most important thing you can take to}

the 95th annual convention and scientific seminar of the

American Osteopathic Association.

November 25-29, 1990

It's United-the airline saving you real money to the American

Osteopathic Association Convention in Las Vegas.

United and United Express now offer special Meeting Plus ${ }^{\mathrm{TM}}$ savings. That means $45 \%$ off regular coach fares. Plus something really unconventional: no minimum length of stay.

Or, if you prefer, we'll give you an additional $5 \%$ off our already low discounted fares, provided you meet the requirements. That could save you as much as $70 \%$ off regular coach fares.

Convening was never this convenient before.

Of course, you'll only be able to get these low fares for travel between $11 / 23-12 / 2 / 90$. All you have to do is provide us with your group's Meeting Plus ${ }^{\mathrm{TM}}$ ID number: 0150J

For reservations, call the United Meetings Plus ${ }^{\mathrm{TM}}$ Desk at 1-800-521-4041. We're open seven days a week from 8 a.m. to 11 p.m. (EST.)

Come fly the friendly skies. 


\section{CHOLESTEROL CONTROL THAT'S CLEARLYUNOUE..}
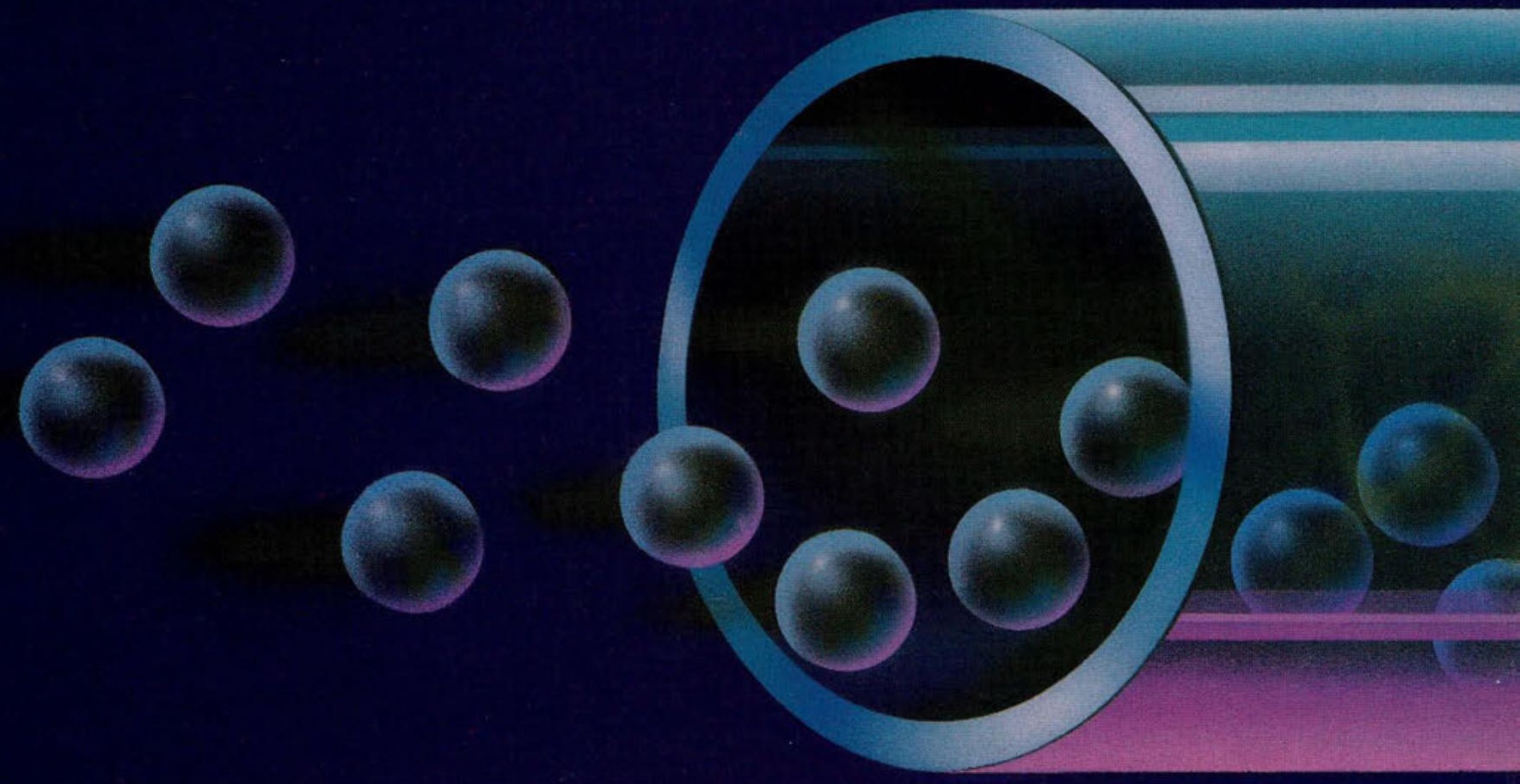

- Unique cholesterol-lowering action -Lorelco ${ }^{\circledR}$ (probucol) enhances the clearance of $L D L$-cholesterol from the bloodstream -increases the removal of cholesterol from the body

\section{- Structurally distinct compound}

- Extensive clinical experience

-maintains lowered levels, as proven over a 12-year period" -well tolerated and convenient for patients to use - just one $500 \mathrm{mg}$ tablet b.i.d. with meals

Lorelco is indicated for the reduction of elevated serum cholesterol in patients with primary hypercholesterolemia, as an adjunct to diet.

Lorelco is not an innocuous drug and strict attention should be paid to the Indications, Contraindications, Warnings, and Precautions sections of Prescribing Information.

- The effect of probucol-induced reduction of serum cholesterol or triglyceride levels or reduction of HDL-cholesterol levels on morbidity and mortality due to coronary heart disease has not been established.

1. Data on File. MERRELL DOW PHARMACEUTICALS INC. Cincinnati. Ohio 45242-9553. 

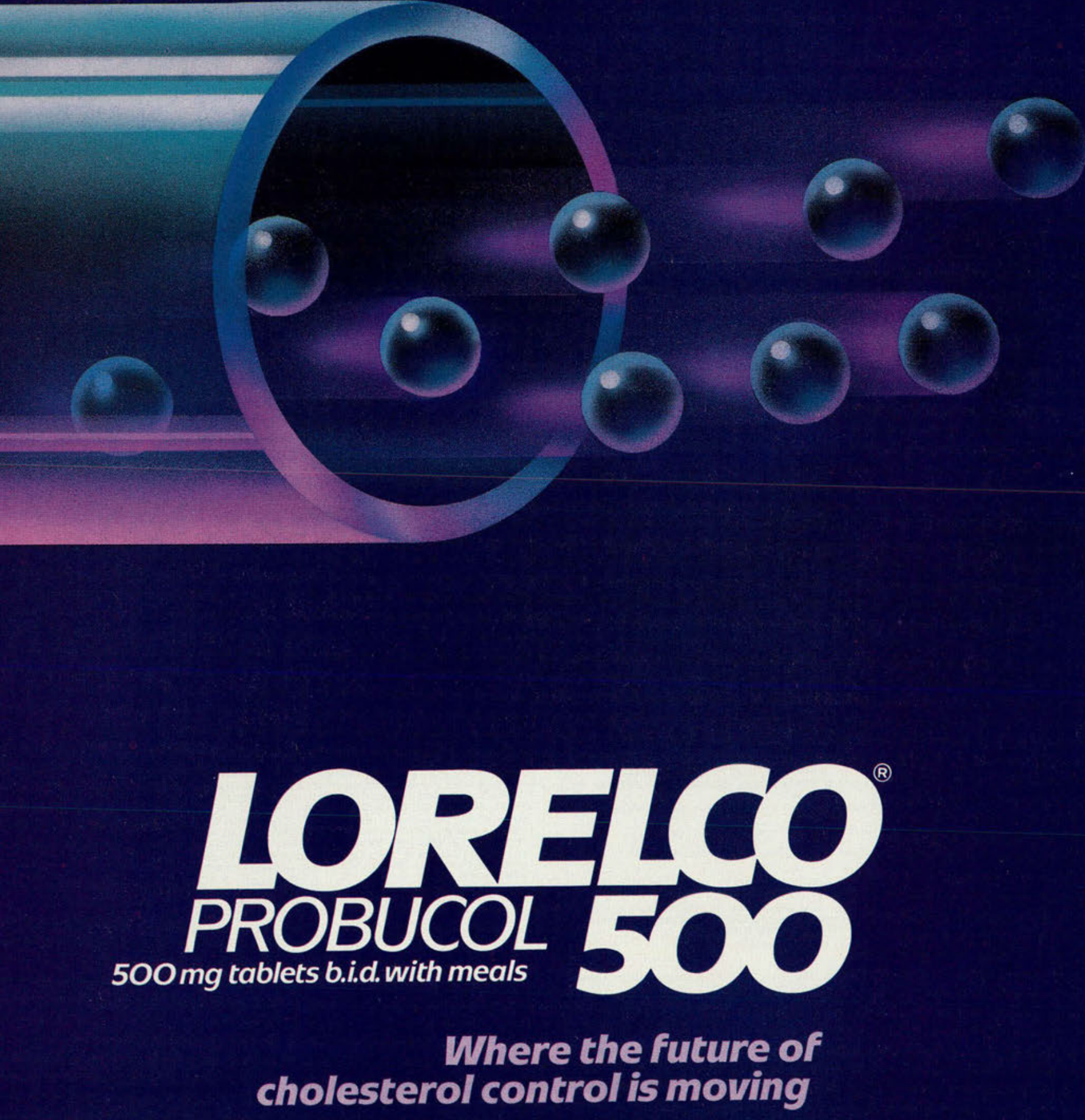


\section{Lorelco ${ }^{\circledast}$ Tablets (probucol)}

CAUTION: Federal law prohibits dispensing without prescription.

DESCRIPTION: Lorelco (probucol) film-coated tablets for oral administration contain $250 \mathrm{mg}$ or $500 \mathrm{mg}$ of probucol per tablet. Each tablet also contains as inactive ingredients: corn starch, ethylcellulose, glycerin, hydroxypropyl cellulose, and titanium dioxide. Lorelco is an agent for the reduction of elevated serum cholesterol. The chemical name is $4.4^{-}-1(1-$ methylethylidene)bis(thio)]bis[2,6-bis(1,1-dimethylethyl)phenol], Its chemical structure does not resemble that of any other available ch

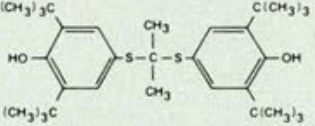

CLINICAL PHARMACOLOGY: Lorelco lowers total serum cholesterol and has relatively little effect on sarum triglycerides in the $\mathrm{LDL}$ fraction, but also in the hight-density lipoprotein ( $\mathrm{HDL}$ ) fraction with proportionately greater effect on the high-density

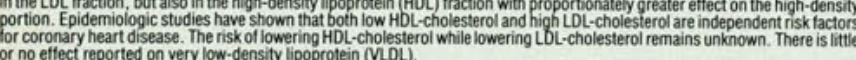

Studies on the mode of action of Lorelco indicate that it increases the fractional rate of $L \mathrm{~L}$ catabolism. This effect may be linked to the observed increased excretion of fecal bile acids, a final metabolic pathway for the elimination of cholesterol from the body.
Lorelco also exhibits inhibition of early stages of cholesterol biosynthesis and slight inhibition of absorption of dietary cholesterol. There is no increase in the cyclic precursors of cholesterol, namely desmosterol and 7 -dehydrocholesterol. On this

Absorption of Lorelco from the gastrointestinal tract is limited and variable. When it is administered with food, peak blood levels are higher and less variable. With continuous administration in a dosage of $500 \mathrm{mg}$. 1. . , the blood levels of an individual
gradually increase over the first three to four months and thereatter remain fairly constant. In 116 patients treated with Lorelco for periods of three months to one year, the mean blood level was $23.6 \pm 17.2 \mathrm{mcg} / \mathrm{mL}( \pm S .0$.) ranging to $78.3 \mathrm{mog} / \mathrm{mL}$
Levels observed after seven years of treatment in 40 patients yielded an average value of $21.5 \pm 16.5 \mathrm{mcg} / \mathrm{mL}( \pm S .0$.) ranging to $62.0 \mathrm{mcg} / \mathrm{mL}$. In a separate study in eight patients, blood levels averaged $19.0 \mathrm{mco} / \mathrm{mL}$ at the end of $12 \mathrm{months}$ of tre

In December 1984, a National Institutes of Health Consensus Development Conference Panell concluded that lowering definitely In December 1984, a National Institutes of Health Consensus Development Conference Paneli concluded that lowering definitely
elevated blood chiolesterol levels (specifically blood levels of $L O L$-cholesterol) will reduce the risk of heart attacks due to coronary heart disease. The effect of probucol-induced reduction of serum cholesterol or trighyceride levels, or reduction of HDL-cholesterol levels on morbidity or mortality due to coronary heart disease has not been established.

INDICATIONS AND USAGE: Serious animal toxicity has been encountered with probucol. See WARNINGS and ANIMAL
PHARMACOLOGY AND TOXICOLOGY sections. Probucol is not an innocuous drug and strict attention should be paid to the INDICATIONS, CONTRAINDICATI

Drug therapy should not be used for the routine treatment of elevated blood lipids for the prevention of coronary heart disease. Dietary therapy specific for the type of hyperlipidemia is the initial treatment of choice. Excess body weight may be an important
tactor and should be addressed prior to any drug therapy. Physical exercise can be an important ancillary measure. Contributory tactor and should be addressed prior to any drug therapy. Physical exercise can be an important ancillary measure. Contributory
disease such as hypothyroidism or diabetes mellitus should be looked for and adequately treated. The use of drugs should be considered only when reasonable attempts have been made to obtain satisfactory results with nondrug methods. If the decisc
ultimately is to use drugs, the patient should be instructed that this does not reduce the importance of adhering to diet. The selection of patients for cholesterol-lowering drug therapy should take into account other important coronary risk factors
such as smoking, hypertension, and diabetes mellitus. Consideration should be given to the efficacy saftery, and compliance such as smoking, hypertension, and diabetes melilitus. Consideration should be given to the efficacy, safety, and comp
factors for each of the cholesterol-lowering drugs prior to selecting the one most appropriate for an individual patient. Lorelco may be indicated for the reduction of elevated serum cholesterol in patients with primary hypercholesterolemia (Types la and Iib hyperl poproteinemia), ${ }^{2}$ whose elevated LDL-cholesterol has not responded adequately to diet, weight reduction, and
control of diabetes mellitus. Lorelco may be useful to lower elevated LDL-cholesterol that occurs in those patients with indicated where hypertriglyceridemia is the abnormality of most indicated where hypertriglyceridemia is the abnormality of most concern. After establishing that the elevation in serum total have an elevaled LDL-chiolesterol as the cause for an elevated total serum cholsesterol. This may be particularly relevant for
patients with elevated triglycerides or with markedly elevated $\mathrm{HDL}$-cholesterol values, where non-LDL fractions may contribute patients with elevated triglycerides or with markedly elevated $\mathrm{HDL}$-cholesterol values, where non-LL fractions may contribute
significantly to total cholesterol levels without apparent increase in cardiovascular risk. In most patients, LDL-cholesterol may
be estimated according to the following equation:

LDL-cholesterol $=$ Total cholesterol $-[(0.16 \times$ triglycerides $)+$ HDL-cholesterol $]$

When total triglycerides are greater than $400 \mathrm{mg} / \mathrm{dL}$, this equation is less accurate. In such patients, LDL-cholesterol may be

It is not always possible to predict from the lipoprotein type or other factors which patients will exhibit favorable results. Lipid

The effect of probucol-induced reduction of serum cholesterol or triglyceride levels, or reduction of HDL-cholesterol levels on

CONTRAINDICATIONS: (See also WARNINGS and PRECAUTIONS.) Lorelco is contraindicated in patients who are known to have a hypersensitivity to it. Lorelco is contraindicated in patients with evidence of recent or progressive myocardial damage or findings suggestive of serious ventricular arthythmias or with unexplained syncope or syn.
Loreico is contraindicated in patients with an abnormally long QT interval.

WARNINGS: SERIOUS ANIMAL TOXICITY HAS BEEN ENCOUNTERED WITH PROBUCOL IN RHESUS MONKEYS FED AN

Prolongation of the QT interval can occur in patients on Lorelco. Serious arrhythmias have been seen in and a concomitant antiarrhythmic drug. The following precautions are deemed prudent

1. Patients should be advised to adhere to a low cholesterol, low fat diet at the start of treatment with Lorelco and throughout the 2. An ECG should be done prior to starting treatment and repeated at appropriate intervals during treatment. If an abnormally
long QT interval is observed, the possible benefits and risks should be carefully considered before making a decision to

Lorelco therapy should be discontinued or not started if the QT interval at an observed heart rate on a resting ECG is OT Interval in $\sec (15 \%$ above the

\begin{tabular}{ccc} 
Observed Heart Rate & \multicolumn{2}{c}{ upper limit of normal) } \\
\cline { 2 - 3 } (beats/min) & Males & Females \\
\hline 40 & 0.56 & 0.58 \\
50 & 0.52 & 0.53 \\
60 & 0.49 & 0.50 \\
70 & 0.45 & 0.47 \\
80 & 0.43 & 0.44 \\
86 & 0.42 & 0.43 \\
92 & 0.40 & 0.41 \\
100 & 0.39 & 0.40 \\
109 & 0.37 & 0.38 \\
120 & 0.36 & 0.36 \\
133 & 0.34 & 0.35
\end{tabular}

272 (Table 6).

3. Patients developing unexplaineds
should have ECG surveillance.

4. Drugs that prolong the $\mathrm{QT}$ interval are more likely to be associated with ventricular tachycardia after:

a. An increase in the dose of the drug.
b. Addition of a second drug that prolongs the QT interval (including tricyclic antidepressants, class I and III antiarthythmics,

c. Hypokalemia or hypomagnesemia.

. Severe bradycardia due to intrinsic heart disease or drug effects on the atrial rate (beta-blockers) or AV block (digoxin).

The use of Lorelco in patients receiving any of these drugs should be based on the conclusion that The use of
alternate methods of hypocholesterolemic therapy are either ineffective or not tolerated, and the poten-
tial benefits of cholesterol lowering outweigh the risk of serious arrhythmia.

The following conditions should be resolved or corrected prior to initiation of therapy with Lorelco.

a. Hypokalemia

Hypomagnesemia
Recent or acute myocardial infarction, ischemia, or inflammation.

PRECAUTIONS General: Before instituting therapy with Lorelco, adequate baseline studies should be performed to determine that the patient cholesterol is not due to secondary conditions such as hypothyroidism, poorly controlled diabetes mellitus, obstructive liver disease, nephrotic syndrome, or dysproteinemias. Serum lipid levels, including HDL-cholesterol, should be determined after an and periodically during combined diet and drug therapy, including assessment during the first preveral monthth of drug treatment atavorable trend in lipid levels should be evident during the first three to four months of administration of Lorelco, and and also lowers $\mathrm{HDL}$-cholesterol in most patients with elevated LDL-cholesterol. Epidemiologic studies within hypercholesterolemic populations have shown that servm $\mathrm{HDL}$-cholesterol is an independendent, inversely correlated, risk factor for
coronary heart disease (see CLINICAL PHARMACOLOGY. Human studies which will attempt to confirm or deny the hypothesis coronary heart disease (see CLINICAL PHARMACOLOGY). Human studies which will attempt to confirm or deny the hypothesis
that drug-induced alteration in HDL-cholesterol affects cardiovascular risk are currently ynder evaluation. It is not known Whether Lorelco-induced reduction of serum HDL-cholesterol will affect cardiovascular risk since no long-term, controlled
clinical trials of Loreico for the prevention of coronary heart disease, similar to the LR-CPPT (see CLINICAL STUDIISS), have been performed. The probable benefits obtained from LOL-cholesterol reduction must be weighed against the possible risk of alteration is not achieved, the drug should be discontinued.
anstion
Information for Patients: The patient should be instructed to adhere to a prudent diet. Fermales should be cautioned agains
becoming pregnant for at least six months after discontinuing Lorelco and should not breast-feed their intants during therapy becoming pre

Laboratory Tests: The physician should schedule periodic blood lipid determinations and periodic ECGs. (See WARNINGS Elevations of the serum transaminases (SGOT. SGPT), bilirubin, alkaline phosphatase, creatine phosphokinase, uric acid. bloof urea nitrogen, and blood glucose above the normal range were observed on one or more occasions in various patients treate therapy. Although the basis for the relationship between Lorelico and these abnormalities is not firm, the possibility that some of these are drug related cannot be excluded. In controlled trials, the incidence of abnormal laboratory values was no higher in the
patients treated with Lorelco than in the patients who received placebo. If abnormal laboratory tests persist or worsen, if clinical
signs consistent with the abnormmal laboratory tests develop, or if systemic manifestations occur, Lorelco should be disconDrug Interactions: The addition of clofibrate to Lorelco is not recommended, since the lowering effect on mean serum level of either $\mathrm{LOL}$ or total cholesterol is generally not significantly additive and, in some patients, there may be a pronounces
lowering of $\mathrm{HDL}$-cholesterol. Neither oral hypoglycemic agents nor oral anticoagulants Monkeys fed a high fat, high cholesterol diet admixed with probucol exhibited serious toxicity. (See WARNINGS and ANIMA
PHARMACOLOGY AND TOXICOLOGY sections.) Prolongation of the OT interval can occur in patients on Loreloo and serious arrtythmias have been seen in association with an abnormally long OT interval in patients on Lorelco. The addition of a second drug that prolongs the QT interval (including tricyclic antidepressants, class I and III antiarrhythmics, and phenothiazines) may
increase the risk of serious arrthythmia. (See CONTRAINDICATIONS AND WARNINGS.) Carcinogenesis, Mutagenesis, Impairment of Fertility

In chronic studies, of wo years' duration in rats, no toxicity or carcinogenicity was observed. These results are consistent with Pregnancy

Pregnancy - Category B: Reproduction studies have been performed in rats and rabbits at doses up to 50 times the human
dose, and have revealed no evidence of impaired fertility or harm to the fetus due to probucol. There are, however, no adequate and well-controlled studies in pregnant women. Because animal reproduction studies are not always predictive of human

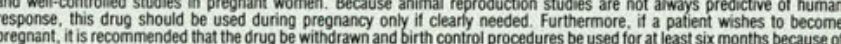
persistence of the drug in the body for prolonged periods. (See CLINICAL. PHARMACOLOGY.)

Labor And Delivery: The effect of Lorelco on human labor and delivery is unknown.

Nursing Mothers: It is not known whether this drug is excreted in human milk, but it is likely, since such excretion has been Pediatric Use: Satety and effectiveness in children have not been established.

ADVERSE REACTIONS

Gastrointestinal

prolionascular of the QT interval on ECG, syncope, ventricular arrtythmias (ventricular tachycardia, torsades de pointes.
ventricular fibrillation), sudden death

Neurologic
headache, diziness, paresthesia, insomnia, tinnitus, peripheral neuritis

Hematologic

eosinophilia, low hemoglobin and/or hematocrit, thrombocytopen

Dermatologic
rash, prurtus, ecchymosis, petechiae, hyperhidrosis, fetid sweat

Genitourinary

Ophthalmic

conjunctivitis, tearing, blurred vision

enlargement of multinodular goiter
End

diosyncrasies

with initiation of therapy and characterized by dizziness, palpitations, syncope, nausea, vomiting and chest pain diminished sense of taste and smell, anorexia, angioneurotic edema

DRUG ABUSE AND DEPENDENCE: No evidence of abuse potential has been associated with Lorelco, nor is there Didence or DSychologicar or physical dependence in humans.

OVERDOSAGE: There is a single report of a $15-\mathrm{kg}$, three-year-old, male child who ingested $5 \mathrm{~g}$ of probucol. Emesis was available on the treatment of overdosage with Lorelco and no specific antidote is available. Probucol is not dialyable. reatment is symptomatic and supportive. Probucol has shown no identifiable acute toxicity in mice and rats. In these DOSAGE AND ADMINISTRATION: For adult use only. The recommended and maximal dose is $1000 \mathrm{mg}$ daily given in HOW SUPPLIED: $250 \mathrm{mg}$ round, white, film-coated tablets imprinted with either the DOW diamond trademark over the $500 \mathrm{mg}$ capsule-shaped, white, film-coated tablets, marked LORELCO 500. Bottles of 100 (NDC 0068-0053-61)

Keep well closed. Store in a dry place. Avoid excessive heat. Dispense in well-closed light-resistant containers with child-

ANIMAL PHARMACOLOGY AND TOXICOLOGY: In rhesus monkeys, administration of probucol in diets containing Premonitory syncope was frequently observed and was associated with a pronounced prolongation of the QT intervals ( 30 to $50 \%$ longer than that observed in untreated monkeys). Serum levels of probucol greater than $20 \mathrm{mcg} / \mathrm{mL}$. were generally Irom control values was usually seen at $40 \mathrm{mcg} / \mathrm{mL}$ and above. Blood levels in humans receiving Lorelco average approximately
$20 \mathrm{mcg} / \mathrm{mL}$ and not uncommonly reach levels of $40 \mathrm{mcg} / \mathrm{mL}$ and higher. Ahesus monkeys ted normal (low fat) chow and receiving probucol three to thirty times the human dose equivalent achieved blood levels only one-third those of many human subjects. No adverse effects were detected in these monkeys over an eight-year period of continuous drug administration. In another study in mesus monkeys, an atherogenic diet was fed for two years and daly treatment with probucol, separated in time
from the atherogenic meal, was carried out during the second year. Serum probuco levels ranged 20 to $50 \mathrm{mog} / \mathrm{mL}$ in five of ten Irom the atherogenic meal, was carried out during the second year. Serum probucool levels ranged 20 to $50 \mathrm{mog} / \mathrm{mL}$ in five of ten
monkeys, and less in the remaining animals. Marked prolongation of the Of interval in the ECG or syncopal behavior was never
observed over the entire one-year treatment period. Regression of gross aortic lesions comparable to that observed in a paralle group of monkeys receiving cholestyramine was seen in animals receiving probucol. It should be emphasized that both HOL. cholesterol and (DL-cholestercl were markedly reduced in this regression s

Subsequent experiments have indicated that probucol sensitizes the canine myocardium to epinephrine, resulting in ventricular fibrillation in many dogs. Among the animal species in which probucol has been studied, the dog is peculiar with respect to the
phenomenon of sudden death due to the sensitization of the myocardium to epinephrine. In contrast to findings in the dog.
injections of epinephrine to probucol-treated monkeys did not induce ventricular fibrillation. In other studies, monkeys were given probucol either before and after, or only after myocardial intarction was induced by
coronary artery ligation. In these studies, there was no difference between probucol-and placebo-treated groups with respect to either survival or detailed blind quantitation of myocardial changes (gross and histopathologic).

Probucol has shown no identifiable toxicity in mice and rats. In these animals, the LDso (oral) is in excess of $5 \mathrm{~g} / \mathrm{kg}$ of body
weight. In chronic studies of two years' duration in rats, no toxicity or carcinogenicity was observed. From studies in rats, dogs, and monkeys, it is known that probucol accumulates slowly in adipose tissue. Approximately $90 \%$ of probucol administered orally is unabsorbed. For that which is absorbed, the biliary tract is the major pathway for clearance from the body and very little is excreted by way of the kidneys.

Myocardial injury was produced in various groups of rats by one of the following procedures: aortic coarctation, coronary
ligation, or cobalt or isoproterenot injection. After probucol administration, no deleterious effects related to treatment occurred sas measured by survival and microscopic examination of myocardial damage. Probucol was administered to minipigs beginning ten days before ligation of coronary artery and continued for 60 days after
surgery. Challenge with epinephrine at the end of 60 days falied to induce ventricular fibrillation in any of the coronary-ligated,
probucol-treated minipigs.

CLINICAL STUDIES: In a multicenter, randomized, double-blind study, the LRC-CPPT, ' hypercholesterolemic patients treated with an oral bile acid sequestrant (cholestyramine) and a cholesteroirlowering diet experienced average total and LDL-
cholesterol reductions greater than those obtained in the placebo goup treated with diet alone. The cumulative seven-year incidence of the primary end point - combined incidence of definite $\mathrm{CHD}$ death and/or definite nonfatal myocardial intarctionwas $7 \%$ in the cholestyramine group and $8.6 \%$ in the placebo group. This was a $19 \%$ reduction in risk (PYess than 0.05 , single-
tail test) of the primary end point reflecting a $24 \%$ reduction in definite $\mathrm{CHD}$ death and a $19 \%$ reduction in nontatal myocardial

The subjects included in the study were middle-aged men (35-59 years old) with serum cholesterol levels at least $265 \mathrm{mg} / \mathrm{dL}$ and no previous history of heart disease. It is not clear to what extent these findings can be extrapolated to other segments of the The bile acid sequestrant, cholestyramine, was used in the above trial. Caution should be exercised in extrapolating these results thect on HOL-cholesterol, and possibile toxicity. The effect of probucol-induced reduction of selestum cholesterol levelen morbidity or mortality due to coronary heart disease has not been established.

REFERENCES

1. Consensus Development Panel. Lowering blood cholesterol to prevent heart disease. JAMA. 1985: 253:2080-2086.
2. Fredrickson DS, Leyy RI, Lees RS. Fat transport in lipoproteins - an integrated approach to mechanisms and disor Engl J Med. 1967:276:34-44.
3. The Lipid Research Clinics Program. The Lipid Research Clinics coronary primary prevention trial results: I. Reduction in Product Information as of June, 1988

Y3420

MERRELL DOW PHARMACEUTICALS INC 
Northup points out, but at least it is less harmful than most therapeutic agents used today.

I would also like to comment on Dr Pecora's article "Factors influencing osteopathic physicians' decisions to enroll in allopathic residency programs" (JAOA 1990;90:527-533). I agree that the quality of allopathic residency programs is one major reason osteopathic physicians choose to enroll in them. Various individuals-including my two sons who are osteopathic physicians-have brought this fact to my attention over the years.

Using the talents of allopathictrained osteopathic physicians is one method to upgrade the quality of our residency programs. However, those persons in positions of power in the American Osteopathic Association have managed, for years, to alienate these physicians by enacting policies that lack insight or imagination. It is time for these individuals to grow up and join the real world.

FRANCIS E. DAVIS, DO

Daytona Beach, Fla

\section{'General practice' requires changing}

\section{To the Editor:}

I am writing to comment on recent discussions regarding the title of "general practice"(JAOA $1990 ; 90: 479)$. Now is the time for both the American Osteopathic Association and the American College of General Practitioners in Osteopathic Medicine and Surgery (ACGP) to rid themselves of this archaic designation. It no longer reflects the training and expertise of these practitioners. The "Rose is a rose" theory may hold true for flowers; however, the outdated term of "general practice" could well be interpreted as a field of medicine that requires less skill than the specialty of "family medicine."

There can be no argument that since its inception, osteopathic medicine has trained its students in and promoted the concept of family medicine, as exemplified in our motto "Health for the Whole Family." What reason could exist that would cause the profession to adhere to such an inappropriate title? Isn't it time for our "general practitioners" to have a modern title that is better accepted and aptly reflects their skills and training?

There is no doubt in my mind that the term "general practice" should be dropped in favor of the more appropriate "family medicine" designation.

DARRYLL W. BARKSDALE, DO Biloxi, Miss

(Editor's note: Dr Barksdale is certified in General Practice by the American Osteopathic Board of General Practice:)

\section{Need for documenting OMT}

\section{To the Editor:}

It's about time we osteopathic physicians responded to the ongoing need for documenting the things we do in the office, namely osteopathic manipulative treatment (OMT). In his article, "Quality assurance monitoring of osteopathic manipulative treatment" (JAOA 1990;90:427-434), Dr Koss presents another compelling reason for documentation-quality assurance.

With fine clarity, Dr Koss describes the bureaucratic balancing act physicians serving the armed forces must undertake. The threat of disciplinary action has catalyzed medical management personnel in the armed forces to take strong measures to protect their clientele-and themselves. These protective measures predictably have taken physicians away from their doctoring, thus intensifying already crippling medical service shortages.

It is difficult to see how one can think of OMT as dangerous when physicians practicing general medicine are faced with a $36 \%$ rate of adverse reactions to drugs or other procedures among hospitalized patients.

Clearly, the literature reflects that osteopathic manipulation of the upper cervical area differs from that performed by chiropractors as a group. Is this because osteopathic medical colleges teach students that high-velocity technique to the high cervical area is performed only with the region in partial-never fullextension? Perhaps this is why clinical reports are virtually devoid of DO-caused damage in the upper cervical area. Maybe this difference accounts for the fact that osteopathic physicians practicing manipulative medicine exclusively traditionally have the (continued on page 766) 